\title{
SINGULARLY CONTINUOUS MEASURES IN NEVAI'S CLASS $M$
}

\author{
D. S. LUBINSKY
}

(Communicated by J. Marshall Ash)

ABSTRACT. Let $d \nu$ be a nonnegative Borel measure on $[-\pi, \pi]$, with $0<$ $\int_{-\pi}^{\pi} d \nu<\infty$ and with support of Lebesgue measure zero. We show that there exist $\left\{\eta_{j}\right\}_{j=1}^{\infty} \subset(0, \infty)$ and $\left\{t_{j}\right\}_{j=1}^{\infty} \subset(-\pi, \pi)$ such that if

$$
d \mu(\theta):=\sum_{j=1}^{\infty} \eta_{j} d \nu\left(\theta+t_{j}\right), \theta \in[-\pi, \pi]
$$

(with the usual periodic extension $d \nu(\theta \pm 2 \pi)=d \nu(\theta)$ ), then the leading coefficients $\left\{\kappa_{n}(d \mu)\right\}_{n=0}^{\infty}$ of the orthonormal polynomials for $d \mu$ satisfy

$$
\lim _{n \rightarrow \infty} \kappa_{n}(d \mu) / \kappa_{n+1}(d \mu)=1 .
$$

As a consequence, we obtain pure singularly continuous measures $d \alpha$ on $[-1,1]$ lying in Nevai's class $M$.

\section{INTRODUCTION}

Suppose that $d \alpha$ is a nonnegative measure on $\mathbb{R}$ with all power moments finite and with support containing infinitely many points. Let $\left\{p_{j}(d \alpha, x)\right\}_{j=0}^{\infty}$ denote the sequence of orthonormal polynomials for $d \alpha$, satisfying

$$
\int p_{j} p_{k} d \alpha=\delta_{j k} \text {. }
$$

The orthonormal polynomials satisfy the recurrence relation

$$
x p_{n}(d \alpha, x)=a_{n+1} p_{n+1}(d \alpha, x)+b_{n} p_{n}(d \alpha, x)+a_{n} p_{n-1}(d \alpha, x),
$$

where $a_{n}>0, b_{n} \in \mathbb{R}$. We say that $d \alpha \in M=M(0,1)$ if

$$
\lim _{n \rightarrow \infty} a_{n}=\frac{1}{2} \quad \text { and } \quad \lim _{n \rightarrow \infty} b_{n}=0 .
$$

For the significance of Nevai's class $M$ and references to the numerous investigations thereof, see [4], [7], [9], and [10].

Received by the editors September 13, 1989 and, in revised form, December 27, 1989.

1980 Mathematics Subject Classification (1985 Revision). Primary 42C05; Secondary 39A11, 41 A99.

Key words and phrases. Orthogonal polynomials, recurrence relations, Nevai's class $M$, singularly continuous measures. 
The most general simple criterion for a measure $d \alpha$ on $[-1,1]$ to belong to $M$ is due to Rahmanov [12]: If $\alpha^{\prime}>0$ a.e. in $[-1,1]$, then $d \alpha \in M$. However, there are measures $d \alpha$ on $[-1,1]$ with $\alpha^{\prime}=0$ a.e. that belong to $M$, and in fact pure jump measures. Explicit examples were first given by the author in [5] and subsequently by Van Assche and Magnus [6]. Probabilistic existence of such measures was established earlier in [1].

At the NATO conference on orthogonal polynomials held at Columbus (MayJune, 1989), the question was raised whether $M$ contains pure singularly continuous measures: That is, $\alpha:[-1,1] \rightarrow \mathbb{R}$ is continuous, but the set of points of increase of $\alpha$ has Lebesgue measure zero. We answer this question affirmatively in this paper, with a fairly explicit example. It is noteworthy that orthogonal polynomials associated with Julia sets often lead to pure singularly continuous measures. See the papers [2], [3] of the group working with J. S. Geronimo.

Associated with any measure $d \alpha$ on $[-1,1]$ is the measure $d \mu$ on $[-\pi, \pi]$, defined by

$$
\mu(\theta):= \begin{cases}\alpha(1)-\alpha(\cos \theta), & \theta \in[0, \pi], \\ \alpha(\cos \theta)-\alpha(1), & \theta \in[-\pi, 0] .\end{cases}
$$

Note that $\mu$ is odd, and so $\mu(0)=0$. We shall also say that $d \mu$ is even. We extend $\mu$ to $\mathbb{R}$ by the periodicity relation

$$
\mu(s+2 \pi)-\mu(t+2 \pi)=\mu(s)-\mu(t)
$$

for all $s, t \in \mathbb{R}$. Equivalently,

$$
d \mu(s \pm 2 \pi)=d \mu(s)
$$

We shall adopt the above normalization and extension to $\mathbb{R}$, for all measures on $[-\pi, \pi]$, without further mention. Note that conversely, any even $d \mu$ (or, equivalently, odd $\mu$ ) generates a measure $d \alpha$ on $[-1,1]$ via the relation $(1.1)$.

One can define orthonormal polynomials

$$
\varphi_{n}(d \mu, z)=\kappa_{n}(d \mu) z^{n}+\ldots, \kappa_{n}(d \mu)>0, n=0,1,2, \ldots,
$$

satisfying

$$
\frac{1}{2 \pi} \int_{-\pi}^{\pi} \varphi_{n}(d \mu, z) \overline{\varphi_{m}(d \mu, z)} d \mu(\theta)=\delta_{m n}, z:=e^{i \theta} .
$$

For $d \alpha$ and $d \mu$ related by (1.1), it is well known [9], [10], [12] that

$$
d \alpha \in M \text { iff } \lim _{n \rightarrow \infty} \kappa_{n}(d \mu) / \kappa_{n+1}(d \mu)=1 .
$$

Our main result is the following:

Theorem 1. Let $d \nu$ be a nonnegative Borel measure on $[-\pi, \pi]$ with $0<$ $\int_{-\pi}^{\pi} d \nu<\infty$ and with the support of $d \nu$ having Lebesgue measure zero. Then there exist $\left\{\eta_{j}\right\}_{j=1}^{\infty} \subset(0, \infty)$, and $\left\{t_{j}\right\}_{j=1}^{\infty} \subset(-\pi, \pi)$ such that

$$
\sum_{j=1}^{\infty} \eta_{j}<\infty
$$


and such that if

$$
d \mu(\theta):=\sum_{j=1}^{\infty} \eta_{j} d \nu\left(\theta+t_{j}\right), \theta \in[-\pi, \pi]
$$

then

$$
\lim _{n \rightarrow \infty} \kappa_{n}(d \mu) / \kappa_{n+1}(d \mu)=1 .
$$

Corollary 2. There exist pure singularly continuous measures $d \alpha$ on $[-1,1]$, lying in $M$.

Remarks. (a) Note that if $d \nu$ is a pure, singularly continuous measure, then clearly so is $d \mu$. Likewise, a pure jump $d \nu$ leads to a pure jump $d \mu$.

(b) We can give a more explicit description for $d \mu$. For $n \geq 1$, let

$$
\theta_{n l}:=-\pi+\left(l-\frac{1}{2}\right) \frac{2 \pi}{n}, \quad 1 \leq l \leq n,
$$

and let $d \rho_{n}$ be the measure placing a mass of $1 / n$ at each $\theta_{n l}$; that is,

$$
d \rho_{n}:=n^{-1} \sum_{l=1}^{n} d \delta_{\theta_{n l}},
$$

where $d \delta_{t}$ is a Dirac delta (unit mass) at $t$. This is just the measure associated with the usual Chebyshev quadrature for $[-\pi, \pi]$, with shifted abscissas. We shall choose $\left\{n_{j}\right\}_{j=1}^{\infty}$ to be a sequence of positive integers, increasing sufficiently rapidly, and set

$$
d \rho:=\sum_{j=1}^{\infty} j^{-2} d \rho_{n_{j}}
$$

Note that $d \rho$ is a pure jump measure and is even, as each $d \rho_{n_{j}}$ is. Finally, for continuous $f$ from the unit circle to $\mathbb{C}$, we set

$$
\int_{-\pi}^{\pi} f\left(e^{i \theta}\right) d \mu(\theta):=\int_{-\pi}^{\pi} \int_{-\pi}^{\pi} f\left(e^{i s} e^{i t}\right) d \rho(t) d \nu(s) .
$$

Since $d \rho$ is a pure jump measure, we shall see in $\S 2$ that $d \mu$ can be written in the form (1.5). Essentially, $d \mu(\theta)$ is the convolution of $d \nu(\theta)$ with $d \rho(\theta)$, and the latter in a certain sense approximates $d \theta /(2 \pi)$.

(c) As a consequence of Szegö's theory [15],

$$
\lim _{n \rightarrow \infty} \kappa_{n}(d \mu)=\infty \text {. }
$$

However, the proof of Theorem 1 shows that we can ensure that $\kappa_{n}(d \mu)$ increases to $\infty$ arbitrarily slowly.

(d) To obtain the measures of Corollary 2, one starts with a pure singularly continuous measure on $[-1,1]$, for example, the equilibrium measure for the 
usual Cantor ternary set. Then we transform to $[-\pi, \pi]$, using (1.1). Then $d \mu$ of Theorem 1 is clearly singularly continuous, and we shall see in $\S 2$, that it is even and so generates a corresponding measure on $[-1,1]$.

(e) For results on the distribution of zeros of orthogonal polynomials on the unit circle and $[-1,1]$, see $[3,7,8,10,11,13,16,17]$.

\section{Proofs}

Throughout, $P_{n}$ denotes the set of polynomials of degree at most $n$, with complex coefficients, and $P_{n}^{0}$ denotes the monic polynomials of degree $n$. Recall first the well-known identities [15, p. 289]

$$
\kappa_{n}^{-2}(d \mu)=\min _{P \in P_{n}^{0}} \frac{1}{2 \pi} \int_{-\pi}^{\pi}\left|P\left(e^{i \theta}\right)\right|^{2} d \mu(\theta)
$$

and $[5$, p. 519,521 and Equations $(2.3),(2.12)]$ for $P \in P_{n-1}$ and $s \in \mathbb{R}$,

$$
\int_{-\pi}^{\pi}\left|P\left(e^{i s} e^{i \theta}\right)\right|^{2} d \rho_{n}(\theta)=\frac{1}{2 \pi} \int_{-\pi}^{\pi}\left|P\left(e^{i \theta}\right)\right|^{2} d \theta .
$$

In the proof of Theorem 1, we shall use the following lemma:

Lemma 2.1. Let $0<\delta<1$ satisfy

$$
0<\delta \log \frac{1}{\delta}<\frac{1}{2} .
$$

Let $S$ be a compact subset of $[-\pi, \pi]$ of Lebesgue measure less than $\delta$, and let $l$ be a positive integer. Then there exists an integer $n>l$, and $P \in P_{n}^{0}$, such that

$$
\left|P\left(e^{i \theta}\right)\right|<2 \delta, \quad \theta \in S,
$$

and

$$
\frac{1}{2 \pi} \int_{-\pi}^{\pi}\left|P\left(e^{i \theta}\right)\right|^{2} d \theta<1+3 \delta \log \frac{1}{\delta}
$$

Proof. Since $S$ is compact, we can choose a (relatively) open subset $T$ of $[-\pi, \pi]$, of measure less than $2 \delta$, containing $S$, and consisting of finitely many (relatively) open intervals. We may assume that $\pm \pi \in T$. Choose $T_{1}$ to be a (relatively) open subset of $[-\pi, \pi]$ containing $\bar{T}$, of Lebesgue measure less than $4 \delta$ and also consisting of finitely many intervals. Define

$$
w(\theta):= \begin{cases}\delta^{-2}, & \theta \in T, \\ 1, & \theta \in[-\pi, \pi] \backslash T_{1} .\end{cases}
$$

In the finitely many intervals between $T$ and $[-\pi, \pi] \backslash T_{1}$, define $w$ to be piecewise linear. Thus $w$ is continuous on $[-\pi, \pi]$, with $w(\pi)=w(-\pi)$ $\left(=\delta^{-2}\right)$, and it satisfies there a Lipschitz condition. Furthermore,

$$
1 \leq w(\theta) \leq \delta^{-2} \text { in }[-\pi, \pi]
$$


By Szegö's theory [15, p. 302, Equation (12.3.15)],

$$
\lim _{n \rightarrow \infty} \kappa_{n}(w)=\exp \left[-\frac{1}{4 \pi} \int_{-\pi}^{\pi} \log w(\theta) d \theta\right]=: A,
$$

and the orthonormal polynomials $\varphi_{n}(w, z)$ satisfy $[15$, p. 297, Theorem 12.1.3]

$$
\lim _{n \rightarrow \infty}\left|\varphi_{n}\left(w, e^{i \theta}\right)\right| w(\theta)^{1 / 2}=1
$$

uniformly for $\theta \in[-\pi, \pi]$. Now as $\log w=0$ in $[-\pi, \pi] \backslash T_{1}$,

$$
A \geq \exp \left[-\frac{4 \delta}{4 \pi} \log \left(\delta^{-2}\right)\right] \geq 1-\frac{2}{\pi} \delta \log \frac{1}{\delta}
$$

and hence, as (2.3) holds,

$$
A^{-1}<1+\delta \log \frac{1}{\delta}
$$

Then the monic polynomial $\Phi_{n}(z):=\varphi_{n}(w, z) / \kappa_{n}(w)$ satisfies

$$
\begin{aligned}
\lim _{n \rightarrow \infty} & \frac{1}{2 \pi} \int_{-\pi}^{\pi}\left|\Phi_{n}\left(e^{i \theta}\right)\right|^{2} d \theta \\
\quad & \frac{1}{2 \pi} \int_{-\pi}^{\pi} w(\theta)^{-1} d \theta / A^{2} \leq 1 / A^{2}<1+3 \delta \log \frac{1}{\delta},
\end{aligned}
$$

as $w \geq 1$ in $[-\pi, \pi]$. Then, for $n$ large enough, $P:=\Phi_{n}$ satisfies (2.5). Furthermore, uniformly for $\theta \in T \supset S$,

$$
\lim _{n \rightarrow \infty}\left|\Phi_{n}\left(e^{i \theta}\right)\right|=\delta / A<2 \delta
$$

in view of (2.3) and (2.6). So we can satisfy (2.4) as well.

Proof of Theorem 1. We shall define $d \mu$ by (1.10), where $d \rho$ is as at (1.7-9), and $\left\{n_{j}\right\}_{j=1}^{\infty}$ is an increasing sequence of positive integers, chosen inductively. We remark that the definition (1.10) does have the form (1.5); more precisely,

$$
d \mu(\theta)=\sum_{j=1}^{\infty} j^{-2} n_{j}^{-1} \sum_{l=1}^{n_{j}} d \nu\left(\theta-\theta_{n_{j}, l}\right), \theta \in[-\pi, \pi] .
$$

One has to take a little care with the definition of integrals with respect to $d \mu$, because of possible jumps of $\nu\left(\theta-\theta_{n_{i}, l}\right)$ at $\theta= \pm \pi$. However, this can be avoided by using (1.10). To prove (2.7), note that, for continuous $f$ on the unit circle, and $n \geq 1$,

$$
\begin{aligned}
& \int_{-\pi}^{\pi} \int_{-\pi}^{\pi} f\left(e^{i s} e^{i t}\right) d \rho_{n}(t) d \nu(s) \\
& =\frac{1}{n} \sum_{l=1}^{n} \int_{-\pi}^{\pi} f\left(e^{i\left(s+\theta_{n l}\right)}\right) d \nu(s) \\
& =\frac{1}{n} \sum_{l=1}^{n} \int_{-\pi+\theta_{n l}}^{\pi+\theta_{n l}} f\left(e^{i \theta}\right) d \nu\left(\theta-\theta_{n l}\right) \\
& =\int_{-\pi}^{\pi} f\left(e^{i \theta}\right) \frac{1}{n} \sum_{l=1}^{n} d \nu\left(\theta-\theta_{n l}\right)
\end{aligned}
$$


where we have also used the $2 \pi$-periodicity of $f$ and $d \nu$. Thus (2.7) is valid and $d \mu$ has the same form as (1.5).

Let us set $n_{1}:=1$. Assume now, as an induction hypothesis, that for some $k \geq 1$, we have already chosen $n_{1}, n_{2}, \ldots n_{k}$. Then we have already constructed the measure

$$
d \mu_{k}(\theta):=\sum_{j=1}^{k} j^{-2} n_{j}^{-1} \sum_{l=1}^{n_{j}} d \nu\left(\theta-\theta_{n_{j}, l}\right),
$$

which is the $k$ th partial sum of the first series in (2.7). Since $d \nu$ has support of Lebesgue measure zero, so does $d \mu_{k}$. Then we can choose a compact set $S$ of measure $<(k+1)^{-2}$ containing this latter support and, say, consisting of finitely many intervals. The endpoints of those intervals in $(-\pi, \pi)$ may be assumed to lie outside the support. According to the lemma, with $\delta:=(k+1)^{-2}$, we can choose an integer $n_{k+1}>n_{k}$, and $P \in P_{n_{k+1}-1}^{0}$, satisfying

$$
\left|P\left(e^{i \theta}\right)\right|<2(k+1)^{-2}, \theta \in S \text {, }
$$

and

$$
\frac{1}{2 \pi} \int_{-\pi}^{\pi}\left|P\left(e^{i \theta}\right)\right|^{2} d \theta<1+6(k+1)^{-2} \log (k+1) .
$$

Then, irrespective of how the $\left\{n_{j}\right\}_{j=k+2}^{\infty}$ will be chosen, their monotonicity, (1.10), and (2.2) ensure that

$$
\begin{aligned}
\int_{-\pi}^{\pi} \mid & \left.P\left(e^{i \theta}\right)\right|^{2} d \mu(\theta) \\
= & \int_{S}\left|P\left(e^{i \theta}\right)\right|^{2} d \mu_{k}(\theta)+\frac{1}{2 \pi} \int_{-\pi}^{\pi}\left|P\left(e^{i \theta}\right)\right|^{2} d \theta\left\{\sum_{j=k+1}^{\infty} j^{-2}\right\} \int_{-\pi}^{\pi} d \nu(s) \\
\leq & 4(k+1)^{-4}\left\{\sum_{j=1}^{k} j^{-2}\right\} \int_{-\pi}^{\pi} d \nu(s)+\left\{1+6(k+1)^{-2} \log (k+1)\right\} k^{-1} \\
& \times \int_{-\pi}^{\pi} d \nu(s) \quad\left[\text { by (2.8) and (2.9) and choice of } d \mu_{k}\right] \\
\leq & k^{-1} \int_{-\pi}^{\pi} d \nu(s)\left\{1+8(k+1)^{-1}\right\} .
\end{aligned}
$$

Hence, from (2.1),

$$
\kappa_{n_{k+1}-1}^{-2}(d \mu) \leq(2 \pi k)^{-1} \int_{-\pi}^{\pi} d \nu(s)\left\{1+8(k+1)^{-1}\right\} .
$$

On the other hand, since for $P \in P_{n}^{0}$,

$$
\frac{1}{2 \pi} \int_{-\pi}^{\pi}\left|P\left(e^{i \theta}\right)\right|^{2} d \theta \geq 1
$$


we see that

$$
\begin{aligned}
\kappa_{n_{k+1}-1}^{-2}(d \mu) & \geq \inf _{P \in P_{n_{k+1}-1}^{0}} \frac{1}{2 \pi} \int_{-\pi}^{\pi} \int_{-\pi}^{\pi}\left|P\left(e^{i s} e^{i t}\right)\right|^{2}\left\{\sum_{j=k+1}^{\infty} j^{-2} d \rho_{n_{j}}(t)\right\} d \nu(s) \\
& =\inf _{P \in P_{n_{k+1}-1}^{0}}\left(\frac{1}{2 \pi}\right)^{2} \int_{-\pi}^{\pi}\left|P\left(e^{i \theta}\right)\right|^{2} d \theta\left\{\sum_{j=k+1}^{\infty} j^{-2}\right\} \int_{-\pi}^{\pi} d \nu(s)
\end{aligned}
$$

$$
\geq[2 \pi(k+1)]^{-1} \int_{-\pi}^{\pi} d \nu(s) .
$$

In this way, proceeding inductively, we can choose $\left\{n_{j}\right\}_{j=1}^{\infty}$ satisfying (2.10) and (2.11) for $k \geq 1$. Note that, then, for $k \geq 2, n_{k} \leq l<n_{k+1}$ implies that

$$
\begin{aligned}
1 & \leq \kappa_{l}(d \mu) / \kappa_{l-1}(d \mu) \\
& \leq \kappa_{n_{k+1}-1}(d \mu) / \kappa_{n_{k}-1}(d \mu) \\
& \leq(k+1)^{1 / 2} /\left\{(k-1)^{-1}(1+8 / k)\right\}^{-1 / 2} \quad \text { [by (2.10) and (2.11)] } \\
& \rightarrow 1,
\end{aligned}
$$

as $k \rightarrow \infty$. So $(1.6)$ is valid.

Proof of Corollary 2. Let $d \beta$ denote a pure singularly continuous measure on $[-1,1]$. For example, we can choose, on $[0,1], d \beta$ to be the equilibrium measure for the usual Cantor ternary set, and then extend $d \beta$ to $[-1,1]$ so as to be even. Define a measure $d \nu$ on $[-\pi, \pi]$ by

$$
\nu(\theta):= \begin{cases}\beta(1)-\beta(\cos \theta), & \theta \in[0, \pi], \\ \beta(\cos \theta)-\beta(1), & \theta \in[-\pi, 0] .\end{cases}
$$

Obviously, $d \nu$ is singularly continuous, and so $d \mu$ of Theorem 1 inherits this property. It remains to show that $\mu$ is an odd function, so that there is a measure $d \alpha$ on $[-1,1]$ corresponding to $d \mu$. Recall that we established (2.7) in the proof of Theorem 1. Since $\nu$ is continuous, integrating (2.7) from 0 to $x$ yields [recalling the normalization $\mu(0)=0$ from the introduction]

$$
\begin{aligned}
\mu(x) & =\sum_{j=1}^{\infty} j^{-2} n_{j}^{-1} \sum_{l=1}^{n_{j}}\left\{\nu\left(x-\theta_{n_{j}, l}\right)-\nu\left(-\theta_{n_{j}, l}\right)\right\} \\
& =\sum_{j=1}^{\infty} j^{-2} \int_{-\pi}^{\pi}\{\nu(x-s)-\nu(-s)\} d \rho_{n_{j}}(s) \\
& =\int_{-\pi}^{\pi}\{\nu(x-s)-\nu(-s)\} d \rho(s) .
\end{aligned}
$$

This expresses $\mu$ as the convolution of $\nu$ and $d \rho$. Further, since $\nu$ is odd and $d \rho$ is even, we can also write

$$
\mu(x)=\int_{-\pi}^{\pi} \nu(x-s) d \rho(s) .
$$


Then

$$
\begin{aligned}
\mu(-x) & =\int_{-\pi}^{\pi} \nu(-x-s) d \rho(s) \\
& =\int_{-\pi}^{\pi}-\nu(x+s) d \rho(s) \\
& =\int_{-\pi}^{\pi}-\nu(x-s) d \rho(s)=-\mu(x)
\end{aligned}
$$

So $\mu$ is odd, and we can define $\alpha$ by (1.1), normalized, for example, by $\alpha(0)=$ 0 . In view of (1.4), $d \alpha$ will belong to $M$.

\section{REFERENCES}

1. F. Delyon, B. Simon, and B. Souillard, From power pure point to continuous spectrum in disordered systems, Ann. Inst. H. Poincaré Phys. Theor. 42 (1985), 283-309.

2. M. F. Barnsley, J. S. Geronimo, and A. N. Harrington, Almost periodic Jacobi matrices associated with Julia sets for polynomials, Commun. Math. Phys. 99 (1985), 303-317.

3. D. Bessis, J. S. Geronimo, and P. Moussa, Function weighted measures and orthogonal polynomials on Julia sets, Constr. Approx. 4 (1988), 157-173.

4. D. S. Lubinsky, A survey of general orthogonal polynomials for weights on finite and infinite intervals, Acta Appl. Math. 10 (1987), 237-296.

5. __ Jump distributions on $[-1,1]$ whose orthogonal polynomials have leading coefficients with given asymptotic behavior, Proc. Amer. Math. Soc. 104 (1988), 516-524.

6. Al. Magnus and W. Van Assche, Sieved orthogonal polynomials and discrete measures with jumps dense in an interval, Proc. Amer. Math. Soc. 106 (1989), 163-173.

7. A. Maté, P. Nevai, and V. Totik, Asymptotics for the ratio of leading coefficients of orthonormal polynomials on the unit circle, Constr. Approx. 1 (1985), 63-69.

8. H. N. Mhaskar and E. B. Saff, On the distribution of zeros of polynomials orthogonal on the unit circle, J. Approx. Theory (to appear).

9. P. Nevai, Orthogonal polynomials, Mem. Amer. Math. Soc., no. 213, Amer. Math. Soc., Providence, RI, 1979.

10. __ Geza Freud orthogonal polynomials and Christoffel functions. A case study, J. Approx. Theory 48 (1986), 3-167.

11. P. Nevai and V. Totik, Orthogonal polynomials and their zeros (to appear).

12. E. A. Rahmanov, On the asymptotics of the ratio of orthogonal polynomials, Math. USSR Sbornik 32 (1977), 199-213.

13. E. B. Saff, Orthogonal polynomials from a complex perspective, Orthogonal Polynomials: Theory and Practice (ed. P. Nevai), NATO ASI Series, vol. 296, Kluwer, Dordrecht, pp. 363-393.

14. H. Stahl and V. Totik, $N$ th root asymptotic behavior of orthonormal polynomials, manuscript, Orthogonal Polynomials: Theory and Practice (ed. P. Nevai), NATO ASI Series, vol. 294, Kluwer, Dordrecht, 1990, pp. 395-418.

15. G. Szegö, Orthogonal polynomials, Amer. Math. Soc. Colloq. Publ., vol. 23, Amer. Math. Soc., Providence, RI, 1939, 4th ed., 1975.

16. J. L. Ullman and M. F. Wyneken, Weak limits of zeros of orthogonal polynomials, Constr. Approx. 2 (1986), 339-347.

17. W. Van Assche, Asymptotics for orthogonal polynomials, Lecture Notes in Math., vol. 1265, Springer, Berlin, 1987.

Department of Mathematics, University of the WitwatersRand, P. O. Wits 2050, RePUBLIC OF SOUTH AFRICA 